\title{
Shade Matching in Aesthetic Dentistry - From Past to Recent Advances
}

Smitha $\mathrm{AJ}^{* 1}$, Savitha $\mathrm{PN}^{2}$

${ }^{1}$ Specialist Prosthodontist, Private dental practice, Dubai, UAE

${ }^{2}$ Senior Lecturer, Department of Prosthodontics, Bangalore Institute of Dental Sciences, Bangalore

${ }^{*}$ Corresponding author: Smitha AJ, Specialist Prosthodontist, Private Dental practice, Dubai, UAE, E-mail: dr.smitha.jacob@gmail.com

Citation: Smitha AJ, Savitha PN (2017) Shade Matching in Aesthetic Dentistry - From Past to Recent Advances. J Dent Oral Care Med 3(1): 102. doi: 10.15744/2454-3276.3.102

Received Date: October 05, 2016 Accepted Date: February 22, 2017 Published Date: May 11, 2017

\begin{abstract}
Aesthetic restorative efforts in the formation of beautiful smiles are defined and guided by certain universal principles. An understanding of the science of color and color perception is crucial to success in the ever expanding field of aesthetic restorative dentistry. Just as the disharmony is created by a discordant note in a symphony, the wrong shades can destroy the result and thus, this necessitates the thorough knowledge and understanding of the concept of shade selection.
\end{abstract}

Keywords: Hue, Chroma, Value, Shade selection, Shade matching, Aesthetics

\section{Introduction}

The vivid colors of nature infuse a dash of brilliance into the vibrant landscapes of life. These colors not only bring joy and brightness into our lives, but are elemental to all forms of beauty. In the field of dentistry, it forms an important basis for achieving superior esthetics. It is a challenge for every esthetic dentist to determine and replicate the appearance of teeth as it requires humility, patience and perseverance to mimic nature to its closest sense and form. Color is governed by visual and scientific components and in dentistry, it is communicated on a regular basis but often misunderstood, since every human eye is not capable of perceiving it in a standardized manner [1].

Clinical shade selection involves more than picking a tab from a shade guide and having the restoration of the same color processed. One often wishes it were that simple, unfortunately, that is the extent of understanding that usually accompanies the shade selection procedure.

\section{Discussion}

\section{Shade Guide}

A shade guide serves to accurately determine a tooth shade. A shade guide, based on the three-dimensional Mun $\neg$ sell Color Order System would be a boon to dentistry and to the color matching of ceramo-metal restorations [2]. In the Munsell system, the relationship of any of the color chips to the chips surrounding it is instantly known by geographic location. If the achromatic axis is to the left, any color chip to the right will be more saturated in color, i.e., have a higher chroma. Any chip lie left will have a weaker chroma. Any chip above will be higher in value, and any chip below will be flower in value. Chips on the same level will be equal in value. Hue changes are predictable from the location ahead or behind the chip on the hue circle.

\section{Dental Shade Guides}

Hyashi shade guides: The guide, which has been published in Japan, uses fully controlled paper tabs to represent each equally spaced 125 locations in color space. Although it is not a commercial guide, it is a concept that can serve as a guideline for future guides. This illustration is a representation of the five hues in the shade guide designed by Hayashi and printed on paper. The illustrated Hayashi guide is based on the Munsell Color Order System with Hue steps of 1.25 inter $\neg$ vals, Value steps of 0.5 intervals, and Chroma steps in unit intervals as shown below:

a. Five Hues-8.75 YR to 3.75 Y with 1.25 interval.

b. Five Values for each Hue- 6 to 8 with 0.5 inter 7 vals.

c. Five Chromas for each Hue-I to 5 with unit interval. 
Clark shade guide (Tooth color indicator): A similar guide was developed in porcelain by Clark 60 years ago. There was 60 in the Clark guide. It contain 60 tab - 3 basic hue, 19 value, 6 chroma. According to him, "Value" is the important dimension to control $[4]$.

Spectatone: used 12 hues, but the shade guide had only every other hue represented. The missing hues could be selected by interpolation. Once the closest hue was selected, the viewer had 36 value and chroma variations of this hue. Since there were 6 hues, a total of 256 selection tabs were available, and an additional 256 tabs could be created by interpolation. The system enabled the viewer to move about in the color space to every hue, value, and chroma needed to achieve the closest match to the tooth being repli-cated. Even though the initial consideration of 256 tabs seemed overwhelming, the guide was simpler and more effective than the illogically ordered sys $\neg$ tems having fewer tabs [5].

VITA Shade guide (VITAPAN CLASS-I): Introduced in 1956, it is a very popular shade guide (Figure 1). Tabs of similar hue are grouped into letter groups like: A (hue of red-yellow) - A1, A2, A3, A3.5, A4 B (hue of yellow) - B1, B2, B3, B4 C (hue of gray) - C1, C2, C3, C4 D (hue of red-yellow-gray) - D2, D3, D4 Chroma is designated with numerical values 1, 2, 3 and 4 (Figure 2).

\section{Limitations:}

a. Not uniformly positioned throughout tooth color space.

b. No standard incremental difference between adjacent shades.

c. In between shades (A2.5) are inaccurate.

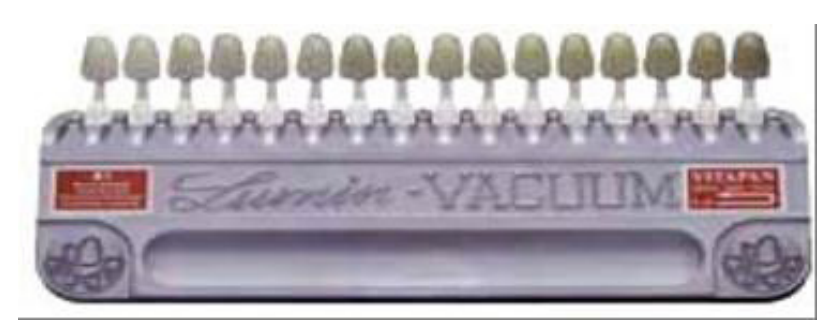

Figure 1: VITA Lumin Shade guide

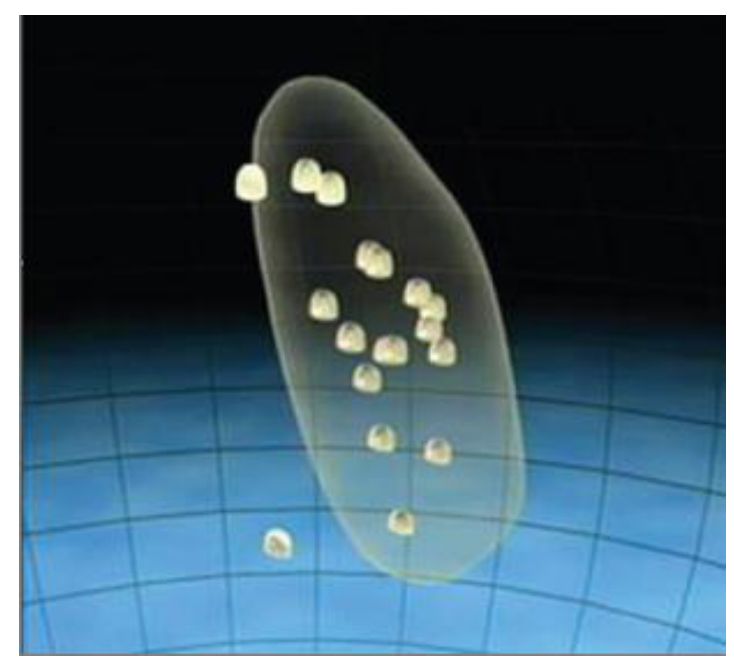

Figure 2: Shade selection with VITAPAN Classic

Vitapan 3D-Master Shade Guide: The manufacturer of this recently introduced shade system claims that it covers the entire color space (Figure 3). It was introduced in 1998 and reflects distribution of tooth shades in nature. There is systematic and equidistant coverage of the natural tooth shade spectrum [6,7].

The shade sample are grouped in six lightness levels each of which has chroma variations in evenly spaced steps. The shade is spaced in steps $(\triangle \mathrm{E})$ of CIELAB 4 units in the lightness dimension and 2 CIELAB units in the hue and chroma dimensions [8]. Because the guide is evenly spaced, intermediate shades can be predictably for $\neg$ mulated by combining porcelain powders. The manufacturer recommends selecting the lightness level first with this system and then selecting the chroma or saturation and finally the hue (Figure 4). A form is available to facilitate the laboratory shade pre $\neg$ scription, which can include intermediate step. Two types of shade guides are available for shade selection in Vitapan 3D master shade guide -

a. Vitapan 3D master tooth guide (blue chips) - vita 3D master tooth guide features fired porcelain shade samples built up with cervical, dentine, incisal powders as known to you from most conventional shade guide.

b. Vitapan 3D master color guide (red chips) - in contrast to Vitapan 3D master tooth guide porcelain sample contain dentin color 
without cervical, incisal distinction used to determine basic body color help to see value, chroma, hue in each third that do not match gradations of color in blue chips.

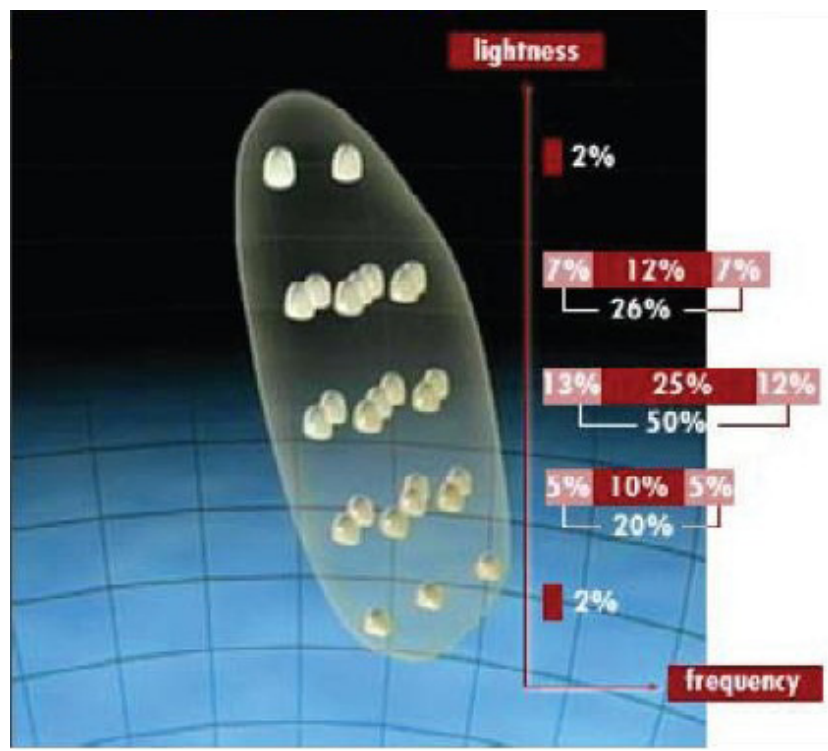

Figure 3: Shade selection with VITAPAN 3D Master Shade Guide

The 3D master is based on the value system rather than grouping the shade by hue as in vita classical and Chromascop lvoclar, Vivodent. The tabs arranged in 5 value level. Within each level tabs present different chroma, hue. Five levels cover that area of the CIELAB color solid occupied by natural teeth, with $50 \%$ of natural tooth shades occupying middle value level. The highest value level has 2 chroma steps of single hue $[9,10]$. Darkest value level has 3 chroma steps of one hue about $2 \%$ natural teeth occupy this.

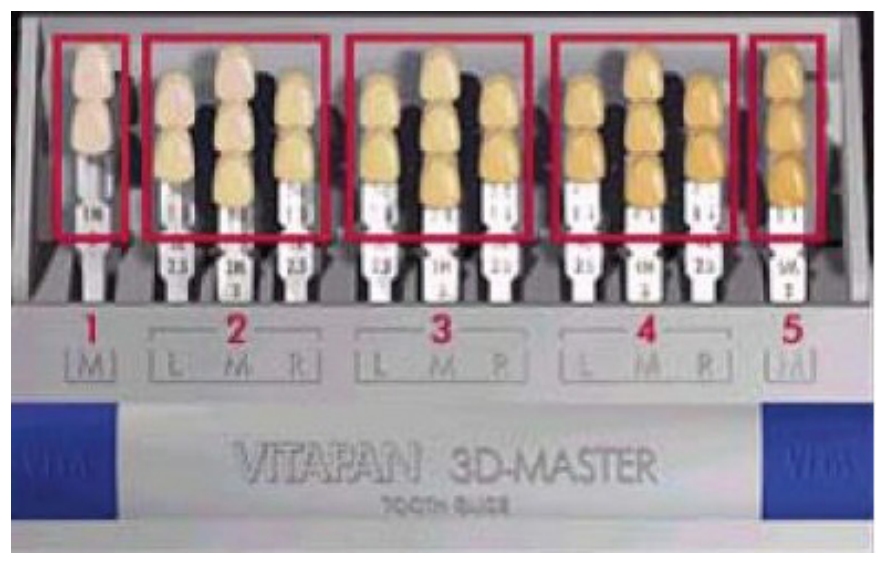

Figure 4: VITA Lumin Shade guide

Visually optimal shade guide: Analoui., et al. (2004) designed an optimal shade guide with the use of a hierarchical technique [11]. The hierarchical clustering is a mathematical procedure for creating a sequence of partitions with in a data set. In this approach the similarity between all tooth samples in the population is computed. With the use of this hierarchical clustering approach a series of shade guide was designed with varying number of tabs. The average error $(\Delta \mathrm{e})$ between colors from each shade guide and the extracted teeth was computed. It was demonstrated that a hierarchical clustering can be used to design an optimal shade guide.

a. Dentin shade guides - When using a translucent all-ceramic system for a crown or veneer communicating the shade of the prepared dentin to the dental laboratory is helpful [12]. One system provides specially colored die materials that match the dentin shade guide and enable the technician to judge restoration esthetics.

b. Custom shade guide - Unfortunately, certain teeth may be impossible to match to commercial shade samples. In addition difficulties may be encountered in reproducing the shade guides in the final restorations. The extensive use of surface staining has severe drawbacks, because the stains increase surface reflection and vent light from being transmitted through porcelain. One approach to this problem is to extend concept of a commercial shade guide by making custom shade guide [13]. An almost infinity number of samples can be made by using different Combinations of porcelain powders in varying distributions. However, the procedure is time consuming and is generally confined to specialty practice.

The fabrication of a custom shade guide, especially one having an expanded shade range can be very helpful. Although fabrication 
of such a guide is time consuming it provides a more realistic representation of what is achievable. Unlike most shade guides, a custom guide is made of the same material as the final restoration, thus reducing metamerism. Miller has recommended the addition of red (pink) modiᄀfiers to supplement the conventional guide in this area of the color space where such guides are lack ᄀing [14]. Fabrication of a custom guide should include a metal backing for metal ceramic restorations, and should be of realistic thickness, achievable with clin-ical restorations. Guides having varying textures and gloss may also be helpful.

c. Modified shade guide - When a tooth closely approximates a specific shade selection tab, but has characterizations or devia $\neg$ tions, those variations may be defined and commu $\neg$ nicated using a shade guide with the glaze removed and a set of dental surface colorants ("stains"). Air $\neg$ borne particle abrading using aluminum oxide is recommended to remove the glaze although this may also be done using emery discs. The colorant may be applied, and removed or modified until the proper effect is achieved $[15,16]$. Once the guide closely resembles the tooth to be matched, it should be placed in a vial to avoid smearing, and sent to the laboratory along with a description of the colorants used and the effects desired.

\section{Drawback of Shade Guides}

a. The inaccuracy in the name of the shade guides is less a problem than the guides themselves they have historically been a weak link in an orderly; approach to color matching in dentistry.

b. The colors of shade guides, from a given manufacturer, vary from guide to guide.

c. The porcelain for the guide is not necessarily the same as the porcelain used for the restoration.

d. The guides do not duplicate the manner in which porcelain restorations must be constructed (thickness of opaque, thick $\neg$ ness of body and incisal porcelain, metal bonding, etc.).

e. The colors of the guides are illogically arranged and do not cover the volume of color space of the natural teeth.

f. All standard shade tabs are thick $(3-4.5 \mathrm{~mm})$ as compared to a crown $(1-1.5 \mathrm{~mm})$, and are made of synthetic resin

g. Light is reflected and transmitted through a shade guide tab giving it translucency and vitality whereas in the restoration, light is reflected and barely transmitted making it look dense and opaque $[6,9,12,15]$.

\section{Recent Advances $[4,7,17,18]$}

Advances in elec $\neg$ tronic technology have pro $\neg$ vided solutions for many of the current problems in shade selection and color matching in dentistry:

a. Colorimeters

b. Spectrophotometer

c. Digital cameras as filter colorimeters

d. Spectrophotometers and spectroradiometers

All color-measuring devices consist of, a detector, signal conditioner and a software that process the signal in a manner that makes the data usable in the dental operatory or laboratory. Because of the complex rela $\neg$ tionship between these elements, accurate colorimetric analysis is difficult at best.

These devices have been designed to aid clinicians and clinicians in the specification and control of tooth color. The earliest color measuring device designed specifically for clinical dental use was a filter colorimeter. The Chromascan (Sternogold Stamford, Connecticut) was introduced in the early 1980s but enjoyed limited success due to its inadequate design and accuracy. Further development was hindered primarily by lack of resources and commitment on industry's side-the market was too small. Now, with esthetics as a major focus of dental marketing and with the availability of improved color measuring optics, companies are willing to make the investment required to apply advanced technology to the challenge of shade control. Duane RD., et al. (1998) used the CIELAB colorimetric system to study the relationship between instrumentally measured color differences and human observer assessment of color differences in metal ceramic crown. The results indicated that dentist have lower tolerances to color difference that result from variation in red chroma as compared with color difference decreased from yellowness. Acceptability of shade difference depends on chromaticity. Observers were more sensitive and critical of crowns whose color differed in redness than whose color differed to the same extent in yellowness $[6,19]$. Correlations between instrumentally derived color differences and visual assessments of perceptibility and acceptability were strong for crowns differing in yellow chroma, red, but weak for lightness. Threshold for acceptability were lower for metal crown differing in red chroma (1.1 $\Delta$ eunits). Threshold for perceptibility $(0.4 \Delta \varepsilon)$ were lower than acceptability for metal ceramic crown differing in their chroma.

\section{Advantage of Digital shade analysis $[12,15,17,19,20]$}

a. Eliminates the subjec $\neg$ tivity of color analysis and provides exact in $\neg$ formation for laboratory fabrication of the prosthesis.

b. Influence is more objective, can be repeatedly verified

c. Not influenced by external factors like surrounding environ $\neg$ ment 
d. Involves less chair-side time.

e. The quality control aspect is a real advantage. The technician can verify that the color replication process was accurate for the shade requested, and. with the more sophisticated systems, a "virtual try-in" can be accomplished.

f. The reading can be translated to materials that can reproduce those characteristics in the fabricated restorations.

\section{Colorimeters}

Filter colorimeters generally use three or four silicon photodiodes that have spectral correction filters that closely simulate the standard observer functions. These filters act as analog function generators that limit the spectral characteristic of the light that strikes the detector surface. The inability to match the standard observer functions with filters while retaining adequate sensitivity for low light levels is the reason that the absolute of filter colorimeters is considered inferior to scanning device like Spectrophotometers and spectroradiometers [21]. However, because of the consistent and rapid sensing nature, these devices can be precise with differential measurements. This is why they often are used for quality control.

\section{Fiber optic colorimeter}

Burget., et al. (1990) 6 described the advantage of fiber optic colorimeter [22]. Tooth color is caused by volume reflection, that is, passage of incident light through the tooth followed by backward emergence. This passage is concurrent with sideward displacement of photons that, in effect, influence the result of usual instrumental methods of determining tooth color. This problem is overcome by the use of large-field illumination and small-field observation. A fiber-optics colorime $\neg$ ter based on this principle is described. The color observed through two holes in a double box was visually matched by subtractive adjustment of the illuminating color in one box, whereas the other box showed the central part of the tooth diffusely illuminated by illuminant C light. This colorimeter was tested on wet, extracted human incisors in the tooth arch of a phantom-head. Results were compared with a visual standard-strip method and with a conventional spectrophotometer. It was concluded that the fiber-optics colorimeter is a promising instrument, although technical improvement is necessary.

\section{Spectrophotometer}

A spectrophotometer is a device that measures the spectral reflectance of a body (Figure 5). It is a photometer (a device for measuring light intensity) that can measure intensity as a function of the color, or more specifically, the wavelength of light [22,23]. A spectrophotometer is a more complicated instrument and there are several configurations. To get a precise measurement of color, it is advisable to use a spectrophotometer. A spectrophotometer measures the reflectance for each wavelength, and allows to calculate values. The general principle is that a light source is diffracted (that is the various wavelengths are spatially separated by a grating or prism). The various wavelengths pass through an entrance slit and the test sample (in some configurations the sample and entrance slit are reversed). The sample selectively absorbs the various wavelengths of light in varying amounts [24]. The light then passes through another slit, called the exit slit, and impinges upon a detector. The detector converts the light intensity at the particular wavelength into an electrical signal that is amplified and displayed on a screen or traced on a chart (light absorbed versus wavelength). There are many variations on this basic design. For example, in some cases the light passing through the sample is compared to a reference signal that passes through some reference sample -- which may contain just the solvent but no active absorber. Today's spectrophotometers contain monochromators and photodiodes that measure the reflectance curve of a product's color every $10 \mathrm{~nm}$ or less. In short, a colorimeter provides an overall measure of the light absorbed, while a Spectrophotometer measures the light absorbed at varying wavelengths $[23,25]$.

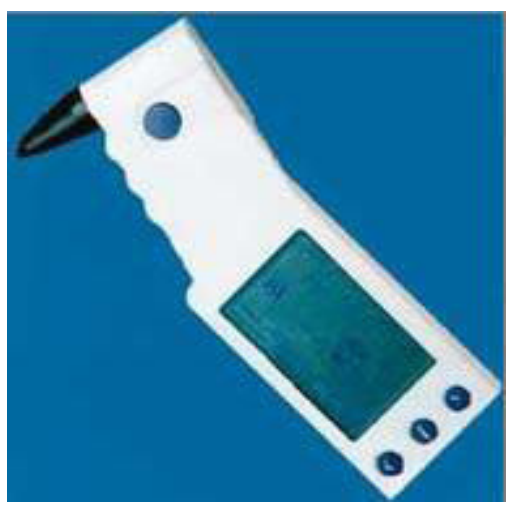

Figure 5: Spectrophotometer

\section{Digital cameras as filter colorimeters}

The newest devices used for dental shade matching are based on digital camera technology. Instead of focusing light upon film to create a chemical reaction, digital cameras capture images using CCDs, which contain many thousands or even millions of 
microscopically small light-sensitive elements (photosites). Like the photodiodes, each photosite responds only to the total light intensity that strikes its surface. To get a full color image, most sensors use filtering to look at the light in its three primary colors in a manner analogous to the filtered colorimeter described previously $[20,26]$. There are several ways of recording the three colors in a digital camera. The highest-quality cameras use three separate sensors, each with a different filter over it. Light is directed to the different filter/sensor combinations by placing a beam splitter in the camera. The beam splitter allows each detector to see the image simultaneously. The advantage of this method is that the camera records each of the three colors at each pixel location.

\section{Spectrophotometers and spectroradiometers}

Are instruments designed to produce the most accurate color measurements. Spectrophotometers differ from spectroradiometers primarily because they include a stable light source. There are two types of basic designs commonly used for these instruments. The traditional scanning instrument consists of a single photodiode detector that records the amount of light at each wavelength [27]. The light is divided into small wavelength intervals by passing through a monochromator. A more recent design uses a diode array with a dedicated element for each wavelength. This design allows for the simultaneous integration of all wavelengths. Both designs are considerably slower than filter colorimeters but remain the tools that are required to examine and develop accurate color-measuring devices.

\section{Currently Available Devices [16,19,25,27,28]}

There are at least six commercially available systems, ranging from simple to complicated, with capabilities and prices to match. The devices are generally one of three types-colorimeters, Spectrophotometers, or digital color analyzers-and use various measuring geometries.
a. Shofu's Shade Chroma Meter
b. The Vita Easyshade
c. The ShadeScan
d. ShadeRite Dental Vision System
e. The SpectroShade
f. ClearMatch System

Shofu's Shade NCC (Natural Color Concept) Chroma Meter (Shofu Dental, Mcnlo Park, California) has been available since the 1990s. It consists of a freestanding, hand-held contact probe that is about $3 \mathrm{~mm}$ in diameter. The probe is placed against the tooth, and an activation button is pushed. This sends a Hash of light to the tooth, from the periphery of the probe, and the reflected light is transported through the center of the probe to the detector where the collected light is evenly distributed through color filters that closely match the three standard observer functions. Data are transmitted to the docking unit via an infrared signal (Figure $6 \mathrm{a}, \mathrm{b}$ ). There is a database of porcelain samples stored in memory, and the closest match of the target with the stored data is presented. Readout is generated that includes the tooth number; the closest Vita Lumin shade guide designation; and specific opaque, body, and enamel powders. Although the ShadeEye was developed for use with the Vintage Halo Porcelain system (Shofu Dental), updated software versions reference other popular porcelains as well [29].
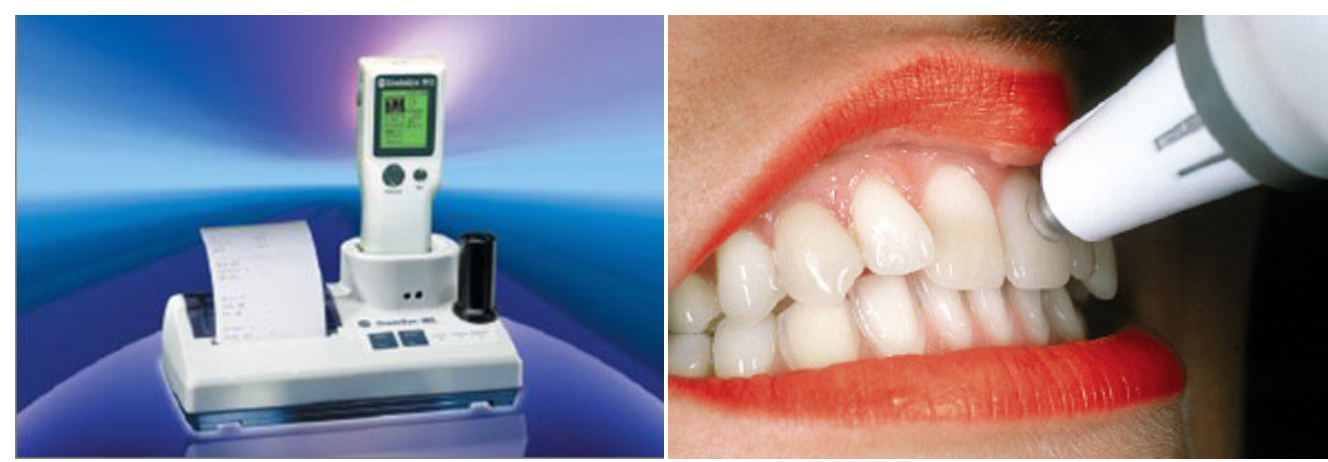

Figure 6a, b: Shofu's Shade NCC (Natural Color Concept) Chroma Meter

The Vita Easyshade (Vident, Brea, California) is a hand-held spectro-photometer that consists of a hand piece connected to a base unit by a monocoil fiber optic cable assembly. The contact probe tip is approximately $5 \mathrm{~mm}$ in diameter (Figure $7 \mathrm{a}, \mathrm{b}$ ).

It contains 19 1-mm-diameter fiber optic bundles. During the measurement process, the tooth is illuminated by the periphery of the tip, directing the light from a halogen bulb in the base unit into the tooth surface. There are several spectrometers in the hand piece that monitor the light source and measure the internally scattered light. A combination of various filters and photodiode arrays receive the light as it is directed through the return fibers located in the center of the probe tip. Through this arrangement, spectral reflectance of the scattered light is essentially measured in $25 \mathrm{~nm}$ bandwidths. Before measurement, it is necessary to select a measurement mode (tooth, crown, or $>$ hade tab). The display presents the closest Vita shade in the classical or 3D shade guide designation [30]. 


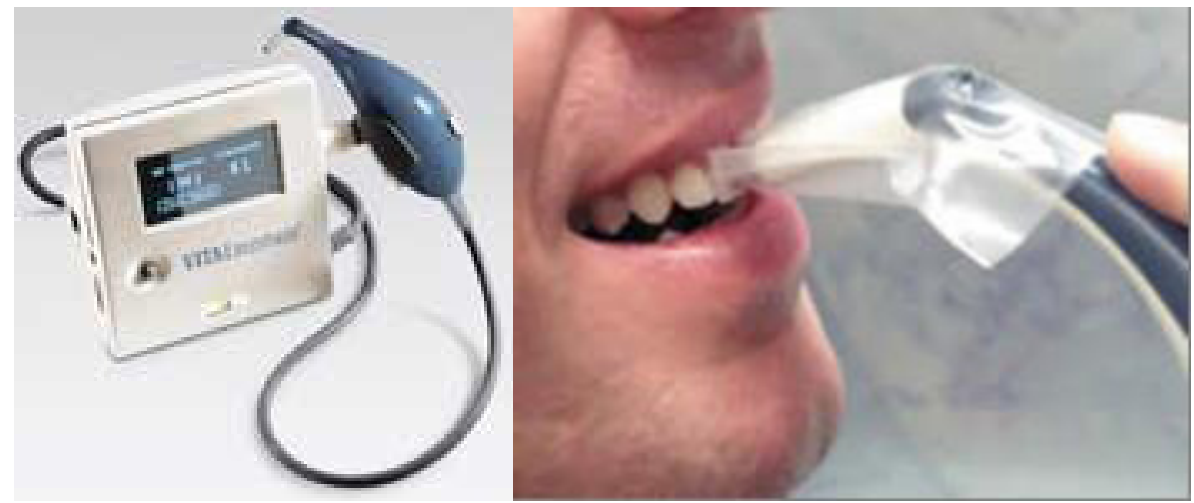

Figure 7a, b: Vita Easyshade

Shade Scan is a hand-held device with a color LCD screen to aid in image location and focus. Through a fiber optic cable, a. halogen light source illuminates the tooth surface at a $45^{\circ}$ angle and collects the reflected light at $0^{\circ}$. Light intensity and calibration to gray and color standards are continuously monitored and adjusted to provide consistent color re $\neg$ production (Figure 8) [31].

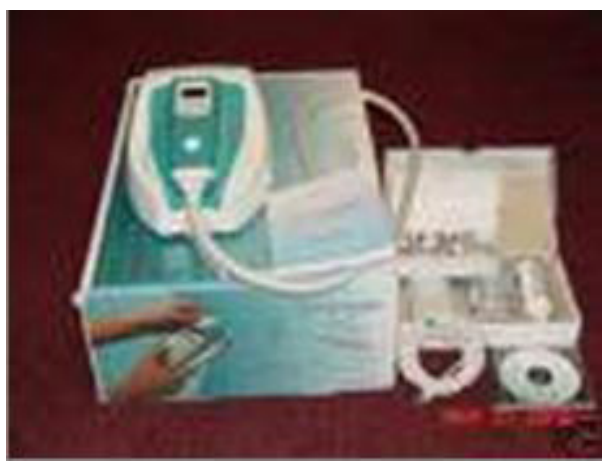

Figure 8: Shade Scan

The image is recorded on a flashcard, obviating the need for a computer in the operatory. The transmitted data can be downloaded to a computer with the Shade Scan software [32]. Shade and translucency mapping can therefore be transmitted to the dental laboratory by e-mail or by including a printout or flashcard with the clinical items required for ShadeScan is in basic Vita Lumin shade designations. Higher-resolution shade mapping, additional shade guide designation conversions, and Hue/Value Chroma values are possible with additional software for dental laboratories [33].

Shade Rite Dental Vision System (X-Rite Inc., Grand Rapids, Michigan) is another instrument that combines digital color analysis with colorimetric analysis (Figure $9 \mathrm{a}, \mathrm{b}$ ). It consists of a hand-held device with its own light source, and an LCD screen facilitates positioning on the tooth.

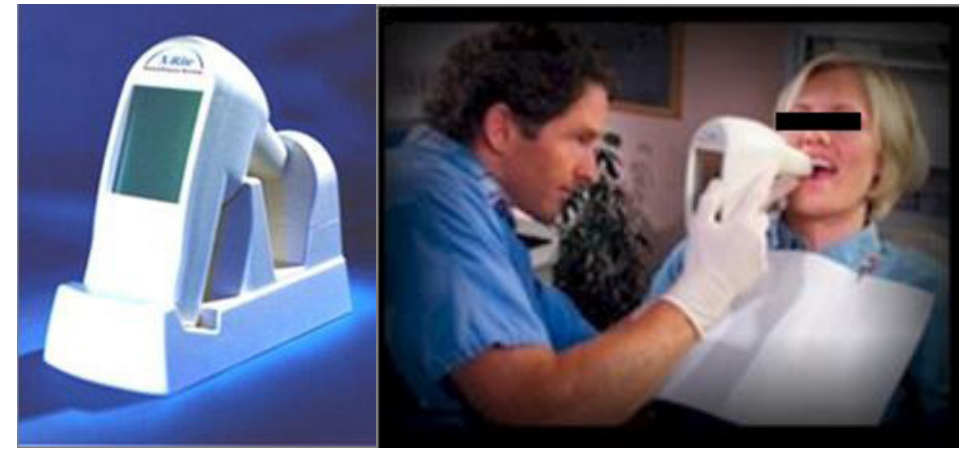

Figure 9: ShadeRite Dental Vision System

To focus and align the camera, a "glare spot" must be located at the junction of the gingival and middle thirds of the tooth. Measurements are taken through a series of rotating filters that simulate the CIE standard observer functions. The device is freestanding and is placed in its docking station for calibration and data transmission to the computer. Shade and translucency mapping, are possible, and colorimetric data (CIE L*a* $\mathrm{b}^{\star}$ values) can be downloaded from the computer. The laboratory must have the required software [30].

SpectroShade (MHT, Niederhasli, Switzerland) is the dental shade-taking device most complex in design and is the most cumbersome in terms of hardware. It offers the most flexibility in terms of color analysis and colorimetric data and is by far 
the most expensive. It is the only one that combines digital color imaging with spectrophotometric analysis. The hand piece is relatively large compared with the contact probe designs, and positioning can be tricky. Calibration is a two-step process involving positioning the hand piece against white and green tiles. Light from a halogen source is delivered through fiber optic bundles and lenses to the tooth surface at $45^{\circ}$. The image of the tooth is displayed on the computer screen so that positioning can be verified. The incident light is monochromatic as it strikes the tooth, and as it is reflected back the spectral scanning process is completed at 10-nm bandwidths by a black and white and a color-filtered CCD. Because there is a spectral curve associated with each pixel of the CCD, a significant amount of data are generated for analysis [30,32]. Color differences can be calculated between compared images, and shade maps of increasing complexity and one for translucency are possible. The software contains shade guide references for most porcelain systems, and mere can be added. The closest shade and the magnitude of the color difference from that reference are specified. A digital image of the tooth, the shade mapping, and the colorimetric data can be transmitted to the laboratory electronically or by printout.

ClearMatch System (Smart Technology, Hood River, Oregon) with this system, a different approach to digital color matching is achieved. This is a software system that requires a Window platform PC and a digital camera. To properly calibrate the digital color signal, a black and white standard and a shade tab must be included in each photograph. Detailed shade mapping is provided in shade guide designations, and standard and custom shade tab information can be entered into the system database. Because this system is software only, it is the most reasonably priced.

\section{Limitations of Digital Shade Analysis [28,31,33]}

a. Accuracy of color measurement is affected by the phenomenon of edge loss.

b. Translucency mapping is inadequate with all of the systems.

c. Positioning of the probe or mouthpiece seems to be critical to the repeatability of the measurement.

d. None of the above instruments are sophisticated enough to function in a formulation mod: (i.e. specifying powders and layering to achieve the actual color designation of any tooth color or translucency distribution measured).

e. For this approach to be efficient the laboratory must have system as well.

f. A few models are avail $\neg$ able in the market, but are very expensive.

\section{Summary}

Selection of proper porcelain shade and color matching ceramic restoration to natural dentition continues to be one of the most perplexing and frustrating problem in fixed prosthodontics. Shade selection forms an important basis for achieving superior esthetics. It is a challenge for every esthetic dentist to determine and replicate the appearance of teeth, as it requires humility, patience and perseverance to mimic nature to its closest sense and form. To provide the patient with an esthetic restoration the dentist must consider the scientific basis of color as well as the artistic aspect. Unlike science, art form is subordinate to the individual abilities of the dentist that influence the predictability and reproducibility of the finished restoration.

There have been a number of recent technologic and material advances that offer the potential to improve color matching in prosthetics.

Media emphasis on an 'esthetic standard' is probably responsible for driving the most recent advances in shade matching. With more research and development it should be possible to achieve a higher percentage of successful match than the approximately $50 \%$ experienced today.

\section{References}

1. Riichiro R, Akirj S, Kanji I (1994) Using a Computer Color-Matching System in Color Reproduction of Porcelain Restorations. Part 3: A Newly Developed Spectrophotometer Designed for Clinical Application. Int I Prosthodont 7: 50-5

2. Schwabacher WB, Richard JG, Marie JL (1994) Interdependence of the hue, value and chroma in the middle site of anterior human teeth. J Prosthod 3: 188-92.

3. Ancovitz, Tony T, Haron R (1998) Texturing and polishing. The final attempt at value control. DCNA 42: 607-12.

4. Duane RD, Jane BD (1998) Acceptability of shade differences in metal ceramic crowns. J Prosthet Dent 79: 254-60.

5. Philip WS, Nairn HW (1998) Shade selection for single-unit anterior metal ceramic crowns: A 5-year retrospective study of 2,500 cases. Int J. Prosthodont 11: 302-06.

6. Scott RO, Ali K, Richards MW, Childress S (1998) Evaluation of visual and instrument shade matching. J Prosthet Dent 80: 642-8.

7. Yap AU (1998) Color attributes and accuracy of Vita-based manufacturers' shade guides. Oper Dent 23:266-71.

8. Duane D, Malgorzata P (1999) Predicting porcelain thickness required for dental shade matches. J Prosthet Dent 82: 143-9.

9. Sato R (1999) Using a computer color matching system in color reproduction of porcelain restoration. Color reproduction of porcelain samples. Int Journal of Prosthodont 6: 522-7.

10. Sato R (1999) Using a computer color matching system in color reproduction of porcelain restoration. A newly developed spectrophotometer for clinical application. Int journal of prosthodont 7: 50-5.

11. Yap AU (1999) Human-eye versus computerized color matching. Oper Dent 24: 358-63. 
12. Akira H, Ikuo I, Satoshi K (2000) Color and translucency of in vivo natural central incisors. J Prosthet Dent 83: 418-23.

13. Minos MS, Efstratios P, Robert RS, William M (2000) Effect of different high-palladium metal-ceramic alloys on the color of opaque porcelain. Johnston and William A. Brantley - J Prosthod 9: 71-76.

14. Yuuji S, Takayasu K (2000) Shade selection for resin-bonded fixed partial dentures. The Journal of Prosthetic Dentistry 83: 527-29.

15. Bruce M (2001) Using tooth and color guides together. The Journal of Prosthetic Dentistry 86: 322-3.

16. Sproul RC (2001) Color matching in dentistry. Part II. Practical application of color. J Prosthet Dent 86: 458-64.

17. Allyson AB, Nicholas JG, Kenneth JA, Mark CY (2002) Influence of tab and disk design on shade matching of dental porcelain. J Prosthet Dent 88: $591-7$.

18. Leila J, Suzanne BR, Rita VM, Pamela BM (2002) Relationship between tooth shade value and skin color: An observational study. J Prosthet Dent 87: 149-52.

19. Rade DP, John MP, Rose-Marie (2002) Color comparison of two shade guides. Int J Prosthodont 15: 73-78.

20. Rade DP (2002) Evaluation of a newly developed visual shade-matching apparatus. Int J Prosthodont 15: 528-34.

21. Dancy WK (2003) Color measurements as quality criteria for clinical shade matching of porcelain crowns. J Esthet Restor Dent 15: 114-21.

22. Hammad IA (2003) Intrarater repeatability of shade selections with two shade guides. J Prosthet Dent 89: 50-3.

23. San P (2003) A shade selection technique. J Prosthet Dent 89: 518-21.

24. Cal E (2004) Application of a digital technique in evaluating the reliability of shade guides. J Oral Rehabil 31: 483-91.

25. John RA, Thomas DT (2004) Vitapan 3D master color guide. DCNA.

26. Mostafa A, Evrika P (2004) Designing visually optimal shade guides. J Prosthet Dent 92: 371-6.

27. Stefanos GK, Aris-Petros T, Asterios AD (2004) Spectrophotometric evaluation of the optical influence of different metal alloys and porcelains in the metalceramic complex. J Prosthet Dent 92: 477-85.

28. Baltzer A (2005) Shading of ceramic crowns using digital tooth shade matching devices. Int J Comput Dent 8: 129-52.

29. Hugo B (2005) Comparison of in vivo visual and computer-aided tooth shade determination. Clin Oral Investig 9: 244-50.

30. Jarad FD (2005) The use of digital imaging for colour matching and communication in restorative dentistry. Br Dent J 199: 43-9.

31. Lee KY, Derric S, Stockes A (2005) Brightness (value) sequence for the vita lumin classic shade guide reassessed. J. Prosthodont rest. Dent 13: 115-118.

32. Wee AG (2005) Clinical color match of porcelain visual shade-matching systems. J Esthet Restor Dent. 17: 351-7.

33. Klemetti E (2006) Shade selection performed by novice dental professionals and colorimeter. J Oral Rehabil. 33: 31-5.

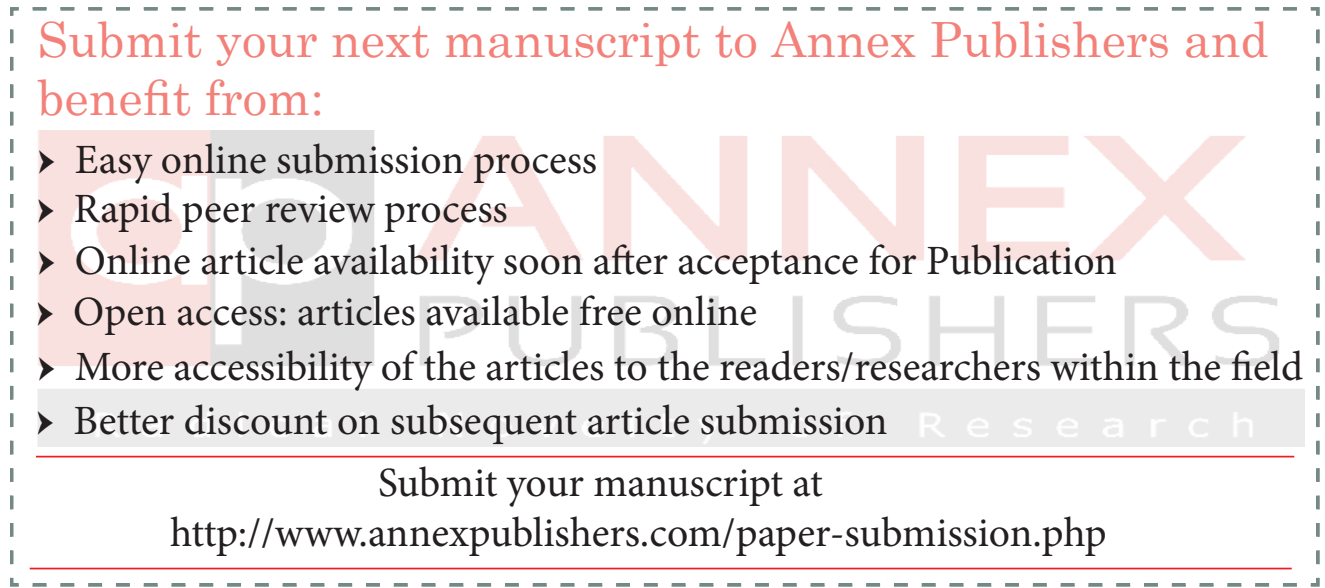

